\title{
The New Strategy for the Treatment of Cerebrospinal Fluid Leak Following Lumbar Surgery
}

\author{
Yuyu Ishimoto ${ }^{12(3)}$, Mamoru Kawakami ${ }^{2}$, Elizabeth Curtis $^{3 / 4)}$, Cyrus Cooper ${ }^{3 / 4)}$, Nami Moriguchi ${ }^{2)}$, Shin-ichi Nakao ${ }^{5)}$ and \\ Yukihiro Nakagawa ${ }^{2}$ \\ 1) Department of Orthopaedic Surgery, Kinan Hospital, Wakayama, Japan \\ 2) Spine Care Center, Wakayama Medical University Kihoku Hospital, Katsuragicho, Japan \\ 3) MRC Lifecourse Epidemiology Unit, University of Southampton, Southampton, United Kingdom \\ 4) Arthritis Research UK/MRC Centre for Musculoskeletal Work and Health, Southampton General Hospital, Southampton, \\ United Kingdom \\ 5) Department of Orthopaedic Surgery, Wakayama Medical University, Wakayama city, Japan
}

\begin{abstract}
:
Introduction: Cerebrospinal fluid (CSF) leak is a common complication of surgery involving the lumbar spine. However, although there are various therapeutic options for CSF leak, there is currently no optimal technique, and the choice of therapy often depends on the surgeon's cumulative experience. The aim of this study was to describe the successful treatment of CSF leakage using blood injection therapy along the drain removal tract.

Technical Note: We enrolled 7 consecutive patients who underwent lumbar surgery at our institute. The surgeries performed included decompression in two patients (one microendoscopic surgery), fusion in four, and an epidural cyst resection in one. After finding a CSF leak, we injected about $10 \mathrm{ml}$ of blood from the patient into the drain tract. CSF leak did not recur after the blood injection in any of the seven patients. Following just one day of bed rest, the symptoms of intracranial hypotension disappeared with no instances of worsened symptoms of back pain, lower limb pain or fever.

Conclusions: We propose this route of blood injection therapy as a novel method for the treatment of CSF leak after lumbar surgery.

Keywords:

Cerebrospinal fluid leak, Lumbar spine, Blood injection, Lumbar surgery, Complication

Spine Surg Relat Res 2020; 4(1): 95-98 dx.doi.org/10.22603/ssrr.2019-0037
\end{abstract}

\section{Introduction}

Cerebrospinal fluid (CSF) leak is a common complication of lumbar spine surgery ${ }^{1-3)}$, causing persistent headaches, nausea, vomiting, and walking difficulties, and it carries an increased risk of meningitis. Although there are various therapeutic options for CSF leak, there is currently no optimal technique. The choice of which technique to perform in each case usually depends on the surgeon's cumulative experience. This study aims to examine the clinical efficacy of a blood injection therapy for CSF leak following lumbar surgery.

\section{Technical Note}

\section{Patients and methods}

We enrolled 7 consecutive patients undergoing lumbar spine surgery at our institution, none of whom suffered from diabetes ( 1 male, 6 female; mean age, 67.4 [range, 49-82] years) (Table 1). The patients and/or their families provided informed consent that data from their case would be submitted for publication. The surgeries performed included decompression in two patients (one microendoscopic surgery), fusion in four, and an epidural cyst resection in one. In five cases, CSF leak from dural tears was noted. In four out of five cases, we sutured the dura and reinforced it using a

Corresponding author: Yuyu Ishimoto, sekitui@wakayama-med-ac.jp

Received: May 10, 2019, Accepted: August 26, 2019, Advance Publication: September 20, 2019

Copyright (C) 2020 The Japanese Society for Spine Surgery and Related Research 
Table 1. Summary of the Patients.

\begin{tabular}{cccllcc}
\hline No. & Gender & Age, year & \multicolumn{1}{c}{ Disease } & \multicolumn{1}{c}{ Operation } & Suturing of dura & Size of tube \\
\hline 1 & F & 67 & LSS & Decompression & No & $19 \mathrm{Fr}$ \\
2 & F & 65 & epidural cyst & cyst resection & Yes & $19 \mathrm{Fr}$ \\
3 & F & 76 & LSS & Fusion+decompression & Yes & $19 \mathrm{Fr}$ \\
4 & F & 82 & LSS & Fusion+decompression & No & $19 \mathrm{Fr}$ \\
5 & F & 66 & LSS & Fusion+decompression & Yes & $19 \mathrm{Fr}$ \\
6 & F & 49 & LSS & Fusion+decompression & Yes & $19 \mathrm{Fr}$ \\
7 & M & 67 & LSS & MEL & No & $10 \mathrm{Fr}$ \\
\hline
\end{tabular}

LSS means lumbar spinal stenosis. Fr means French. MED means microendoscopic laminectomy

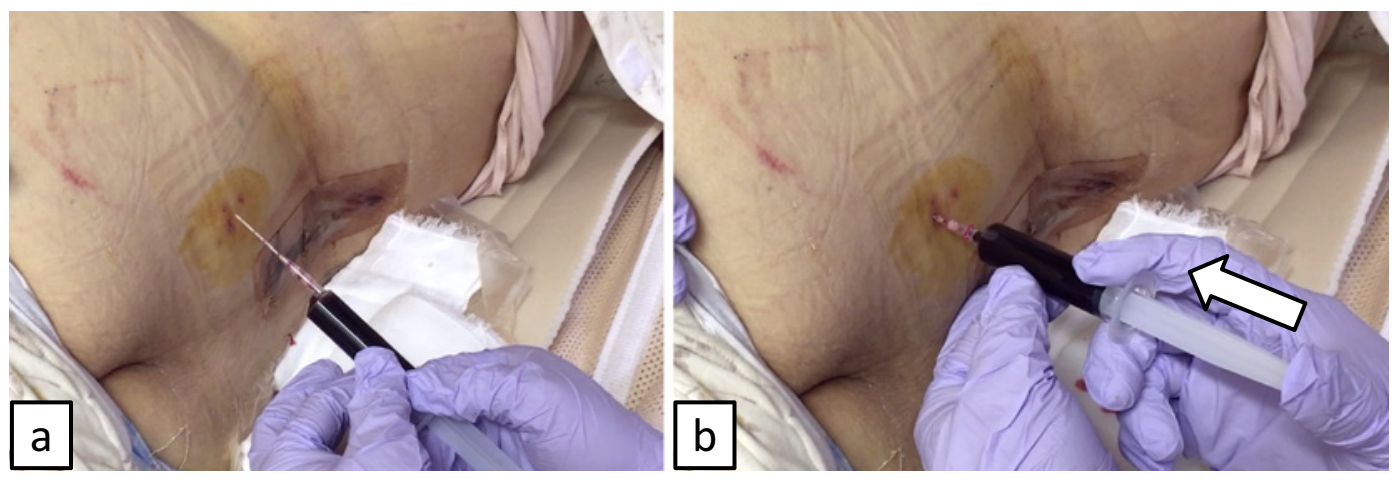

Figure 1. a. An indwelling plastic needle is inserted into the drain tract, and blood from the patient is injected into the needle. b. Blood is injected slowly while checking the patient's clinical state.

small piece of polyglactin 910 (Vicryl Knitted Mesh, Ethicon, Somerville, New Jersey) and fibrin glue (Bolheal, Astellas, Tokyo, Japan) $)^{4}$. In microendoscopic decompression, we used a small piece of polyglactin 910 and fibrin glue without suturing the dura. After repairing the dural tear, we checked the CSF leak using the Valsalva maneuver. We also used soft fluted silicone drain (Blake drains, Ethicon, Somerville, N.J.) ${ }^{5}$ in all surgeries (drain size: 19 French in 6 cases, 10 French in 1 case), and all were drained at the same pressure. In all cases, the lumbar dressings of the patients were saturated with clear fluid (Fig. 1a), and they had intracranial hypotension syndrome with symptoms such as postural headaches caused when moving from a lying to standing position. In addition, we measured the glucose concentration of the exudate using urine dip test paper. We diagnosed CSF leak when the glucose concentration was less than $100 \mathrm{mg} / \mathrm{dl}^{1)}$.

After diagnosing a CSF leak, we performed venesection from the patient's medial cubital vein. We removed the silicone drain and injected about 4-5 $\mathrm{ml}$ of blood into the drain tract with an indwelling needle (Fig. 1a, b) until we saw overflowed blood from the tract. The patient's clinical status was assessed following the blood injection and was based on the presence of the following: evidence of recurrence of CSF leak, intracranial hypotension symptoms, low back pain, lower limb symptoms, and fever.

All patients were advised to take a day of bed rest and all patients could stand and walk reassuringly the next morning. CSF leak did not recur after the procedure in any of the patients. The symptoms of intracranial hypotension resolved, and no complications such as worsening back pain, lower limb pain, or fever occurred.

\section{Discussion}

Our current results demonstrate that this simple procedure for postoperative CSF leaks provides a good alternative to reoperation. All our patients were advised to have a day of bed rest following the blood patch injection, and CSF leak did not recur in any of the patients.

Several methods are used for the management of CSF leak including direct suturing ${ }^{1,6)}$, percutaneous injection ${ }^{7,8)}$, drainage therapy ${ }^{9-11)}$, and blood patch ${ }^{9,12-14)}$. One well-known and widely accepted management option is surgical repair by either meticulous direct primary closure of the dura or augmented closure by means of fat, muscle tissue, or a fascia graft. Although these seem to be the most reliable method for CSF leak, four cases in our study suffered from CSF leak after surgical repair wherein we sutured the injured dura and reinforced it using polyglactin 910 and fibrin glue.

Patel et al. ${ }^{8}$ assessed the effectiveness of CT-guided percutaneous injection of fibrin glue made from patients' blood into the epidural space in a case series of 23 CSF leaks. In 15 of 23 cases, the symptoms were absent or markedly decreased after the procedure and a period of overnight bed rest. However, the complication of aseptic meningitis occurred in five cases. In addition, it took several days to prepare the cryoprecipitate from the patient's blood. Epidural blood patch and drainage therapy were also reported as 


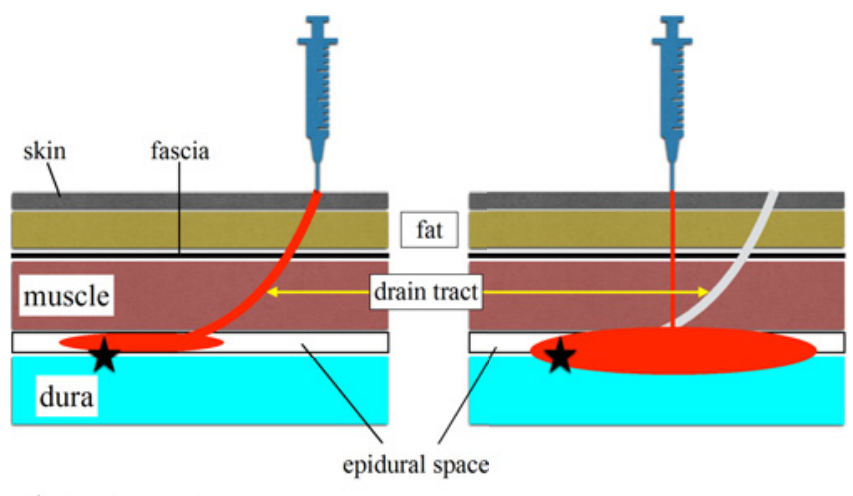

leakage point

left: our method, right: blood patch

Figure 2. Blood injection approaches to seal a CSF leak. Left: the procedure used in this case series. Entering via the drain tract may be the most accurate way of reaching a dural tear, enabling us to seal both the leakage point itself and the route from dura to skin by using less than $10 \mathrm{ml}$ blood. Right: Using the standard epidural blood patch technique, requiring approximately $20-30 \mathrm{ml}$ of blood. In addition, the drain tract is not sealed up with blood

other alternative procedures to surgical closure.

Lauer et al. ${ }^{12}$ reported the effect of epidural blood patch for CSF leak after lumbar decompression surgery. The clinician inserted a Tuohy needle beside the surgical incision and injected $20 \mathrm{ml}$ of the patient's blood to the L4-L5 epidural space. The next day, the patient noted complete resolution of headache and was able to stand without discomfort; however, three days after this procedure, dull back pain occurred, radiating to the left leg. The differences between our procedure and the standard epidural blood patch therapy are shown in Fig. 2, highlighting the fact that it can be difficult to find the epidural space immediately following lumbar surgeries using the standard epidural blood patch procedure. We suggest that by using our drainage tract method, these problems may be overcome.

Shapiro et al. ${ }^{11)}$ reported that the success rate of lumbar subarachnoid catheterization and drainage for 107 CSF leak cases was 94\%; however, complications can occur such as infections $(5 \%)$, occlusion of the drain requiring replacement $(9 \%)$, and transient nerve root irritation (14\%). Jackson-Platt drainage was also used for 16 CSF leaks, though the drain remained in place for 10-14 days and 5 patients needed reoperations. These previously reported methods require a specialist technique, requiring recuperation for several days, and were not successful in all cases.

In our opinion, our proposed method of injecting blood along the drain removal tract may be the most accurate way of reaching the dural tear, sealing both the leakage point itself and the route from the dura to the skin. A benefit of this procedure is that it can be repeated if the blood injection is unsuccessful on the first attempt.

However, various limitations to our proposed approach should be recognized. Although this method may be a useful procedure for treating CSF leak, it must be acknowledged that this is an experimental procedure, a small sample size is reported here, and side effects similar to those seen in epidural blood patch therapy could be expected. Also, although we performed MRI in a few cases at an early stage in the collection of this series to check the size of the dural tear and its site, tears could not be seen on imaging. We noted that there was no pseudomeningocele in these patients, suggesting that large dural tears would not have been present. As we have no post-repair imaging data, we do not know to what extent the blood injection sealed the dural tear. Therefore, in future studies, imaging using injection of contrast medium into the drain tract may provide further information. We had no controlled cases; however, in our first two cases, we waited three days and made them be on bed without standing and sitting. But the 3 days of bed rest did not work for CSF leakage; therefore, we performed this method.

Nevertheless, this is the first report to use the blood injection therapy along the drain removal tract for the treatment of CSF leak, and this method may provide an alternative to surgical closure. In conclusion, our method of blood injection along the drain removal tract was performed without difficulty in seven cases, and following just one day of bed rest, this method was effective in all cases. We propose this route of blood injection therapy as a novel method for the treatment of CSF leak after lumbar surgery.

Conflicts of Interest: The authors declare that there are no relevant conflicts of interest.

Author Contributions: YI wrote and prepared the manuscript, YI and EC contributed to the analysis and interpretation of the results, and EC corrected the English. All authors have read, reviewed, and approved the article.

\section{References}

1. Menon SK, Onyia CU. A short review on a complication of lumbar spine surgery: CSF leak. Clinl Neurol Neurosurgery. 2015;139: 248-51.

2. Patel MR, Louie W, Rachlin J. Postoperative cerebrospinal fluid leaks of the lumbosacral spine: management with percutaneous fibrin glue. AJNR. 1996;17(3):495-500.

3. Guerin P, El Fegoun AB, Obeid I, et al. Incidental durotomy during spine surgery: incidence, management and complications. A retrospective review. Injury. 2012;43(4):397-401.

4. Shibayama M, Mizutani J, Takahashi I, et al. Patch technique for repair of a dural tear in microendoscopic spinal surgery. JBJS. 2008;90(8):1066-7.

5. Niinami H, Tabata M, Takeuchi Y, et al. Experimental assessment of the drainage capacity of small silastic chest drains. Asian Cardiovasc Thorac An. 2006;14(3):223-6.

6. Eismont FJ, Wiesel SW, Rothman RH. Treatment of dural tears associated with spinal surgery. JBJS. 1981;63(7):1132-6.

7. Mihlon F, Kranz PG, Gafton AR, et al. Computed tomographyguided epidural patching of postoperative cerebrospinal fluid leaks. J Neurosurg Spine. 2014;21(5):805-10.

8. Patel MR, Caruso PA, Yousuf N, et al. CT-guided percutaneous fibrin glue therapy of cerebrospinal fluid leaks in the spine after 
surgery. AJR. 2000;175(2):443-6.

9. McCormack BM, Taylor SL, Heath S, et al. Pseudomeningocele/ CSF fistula in a patient with lumbar spinal implants treated with epidural blood patch and a brief course of closed subarachnoid drainage. A case report. Spine. 1996;21(19):2273-6.

10. Hughes SA, Ozgur BM, German M, et al. Prolonged Jackson-Pratt drainage in the management of lumbar cerebrospinal fluid leaks. Surg Neurol. 2006;65(4):410-4.

11. Shapiro SA, Scully T. Closed continuous drainage of cerebrospinal fluid via a lumbar subarachnoid catheter for treatment or prevention of cranial/spinal cerebrospinal fluid fistula. Neurosurgery. 1992;30(2):241-5.

12. Lauer KK, Haddox JD. Epidural blood patch as treatment for a surgical durocutaneous fistula. J Clin Anesth. 1992;4(1):45-7.

13. Maycock NF, van Essen J, Pfitzner J. Post-laminectomy cerebrospinal fluid fistula treated with epidural blood patch. Spine. 1994; 19(19):2223-5.

14. Tanweer O, Kalhorn SP, Snell JT, et al. Epidural Blood Patch Performed for Severe Intracranial Hypotension Following Lumbar Cerebrospinal Fluid Drainage for Intracranial Aneurysm Surgery. Retrospective Series and Literature Review. J Cerebrovasc Endovasc Neurosurg. 2015;17(4):318-23.

Spine Surgery and Related Research is an Open Access journal distributed under the Creative Commons Attribution-NonCommercial-NoDerivatives 4.0 International License. To view the details of this license, please visit (https://creativeco mmons.org/licenses/by-nc-nd/4.0/). 